\title{
A Testbed for Implementing Prognostic Methodologies on Cryogenic Propellant Loading Systems
}

\author{
Chetan S. Kulkarni*, George Gorospe*, Matthew Daigle ${ }^{\dagger}$, and Kai Goebel ${ }^{\dagger}$ \\ ${ }^{*}$ SGT Inc., NASA Ames Research Center, Moffett Field, CA 94035, USA \\ chetan.s.kulkarni@nasa.gov \\ george.gorospe@nasa.gov \\ ${ }^{\dagger}$ NASA Ames Research Center, Moffett Field, CA 94035, USA \\ matthew.j.daigle@nasa.gov \\ kai.goebel@nasa.gov
}

\begin{abstract}
Prognostics technologies determine the health state of a system and predict its remaining useful life. With this information, operators are able to make maintenance-related decisions, thus effectively streamlining operational and missionlevel activities. Experimentation on testbeds representative of critical systems is very useful for the maturation of prognostics technology; precise emulation of actual fault conditions on such a testbed further validates these technologies. In this paper we present the development of a pneumatic valve testbed, initial experimental results and progress towards the maturation and validation of component-level prognostic methods in the context of cryogenic refueling operations. The pneumatic valve testbed allows for the injection of time-varying leaks with specified damage progression profiles in order to emulate common valve faults. The pneumatic valve testbed also contains a battery used to power some pneumatic components, enabling the study of the effects of battery degradation on the operation of the valves.
\end{abstract}

\section{INTRODUCTION}

Prognostics technology is centered on the determination of the health state of a component, subsystem, or system, and the prediction of critical events, such as the end of life (EOL). With prognostics, operators can take informed maintenance decisions to make operational and mission-level activities more optimal, efficient, and cost-effective. In particular, in cryogenic propellant loading operations, launch availability can be maintained and maintenance cost reduced through the use of prognostics and other health management technologies [1]. A cryogenic propellant loading testbed has been developed at NASA Kennedy Space Center (KSC) that transfers cryogenic propellant from a storage tank to a vehicle tank through a network of pipes, pumps, and valves, in order to mature health management technologies for launch support systems [2], [3].

In propellant loading systems, and many others, pneumaticactuated valves play an important role [4], [5]. These valves are used to control propellant flow, and, therefore, failures may have a significant impact on launch availability. In this context, we have developed a pneumatic valve testbed that allows the controlled injection of faults on a subset of valves used in the larger testbed at KSC. Such a testbed allows the demonstration of prognostics for such systems, as well as maturation and validation of the technology.

In addition to valves, the testbed also includes a set of batteries used to power some components in the testbed, allowing the implementation of prognostics for batteries as well. In many launch support systems, batteries are used as a backup power supply sources. In these contexts, it is critical to both monitor battery health and performance and to predict end of discharge (EOD) and EOL events. The testbed allows for the implementation of battery prognostics and the study of the effects of battery degradation on other system components.

In previous work, we described the initial design of the prognostics testbed [6]. Subsequent to this, construction of the testbed was completed, with the testbed being used to control the injection of fault modes on a solenoid valve, a currentpressure transducer, a pneumatic valve that can be controlled only to open and closed positions, and a pneumatic valve that can be controlled to any discreteposition. Additionally, battery health monitoring and predictive methods were implemented on the laboratory testbed. The testbed is able to inject four different leakage faults and one battery fault. This paper presents the final testbed design, discusses the integration with prognostics algorithms, and describes detailed experimental results. We discuss the prognostic results for batteries while the details of implemented prognostics methodologies for pneumatic valves in the testbed are discussed in [5], [7].

The structure of the paper is as follows. Section II discusses the overall design of the prognostics testbed. Section III describes the faults injected in the system. Section IV briefly describes the integration with prognostics algorithms. Section $\mathrm{V}$ discuss the experiments conducted and some illustrative prognostics results. The paper ends with discussion and conclusions in Section VI.

\section{Prognostic Testbed Design}

The testbed as shown in Figure 1 has been designed and developed to simulate valve faults and demonstrate remaining life prediction of valves and batteries in the con- 


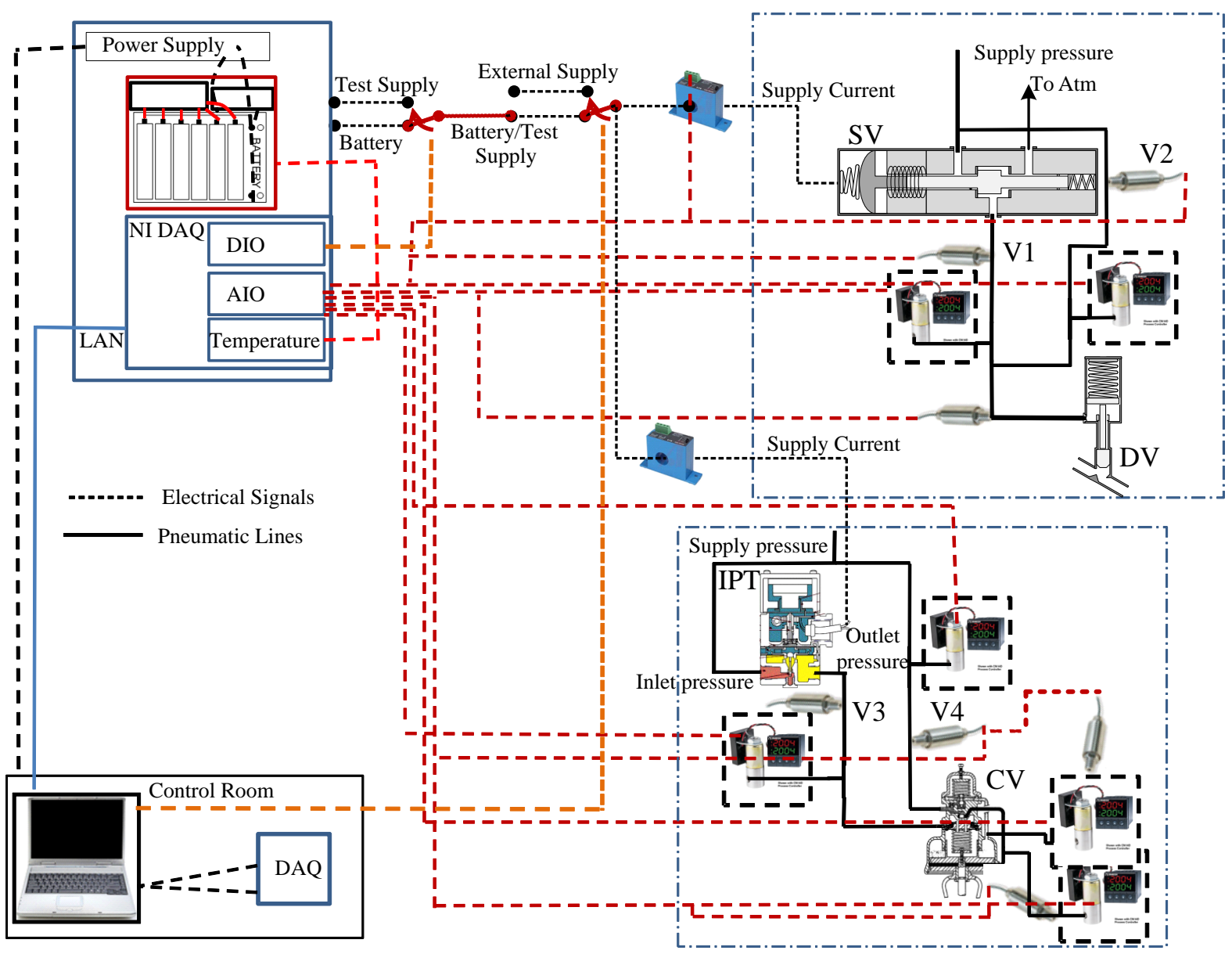

Fig. 1. Schematic for complete Setup

text of cryogenic refueling operations. The solid lines are the pneumatic pressure lines connecting the control valves, solenoids, IPT(Current Pressure Transducer), LVDT (linear variable differential transformer) etc. The dashed lines are the electrical signals to the IPT and solenoid valve. Pressure sensors are placed at specific locations to monitor the supply, signal, control pressures respectively in each of the lines.

Two types of pneumatically-actuated valves are used within the prognostics testbed. The discrete-controlled valve (DV), illustrated in Figure 2, is a normally-closed valve with a linear cylinder actuator. The valve is opened by filling the chamber and piston assembly above the valve with gas up to the actuation pressure, and closed by evacuating the chamber down to atmospheric pressure. When pressure is lost, the valve will close due to the force exerted by the return spring, hence it is a normally-closed valve [4].

The continuous-controlled (CV) valve, illustrated in Figure 3 , on the other hand, opens in a continuous manner. Like the DV valve, the actuator contains a chamber and piston assembly, however, internally, the actuator contains additional components in order to modulate the pressure applied to the piston. The actuator has two pressure ports, one for the supply pressure, and one for the signal pressure. The signal pressure

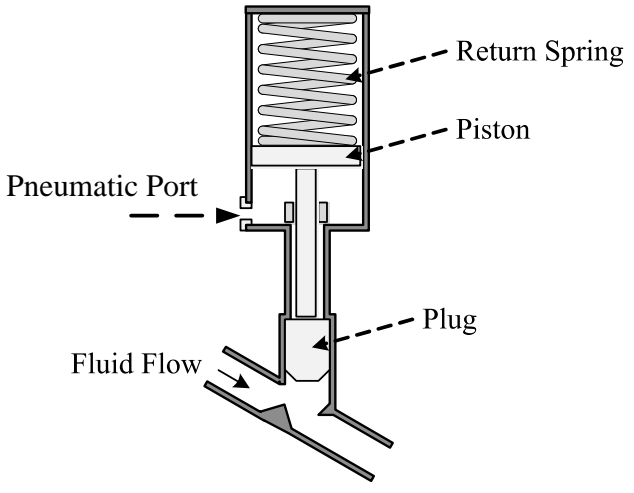

Fig. 2. Discrete-controlled valve

is controlled between 3-15 psig in order to move the valve between fully closed and fully open. The actuator contains a 3-way, spool type pilot valve. Supply pressure is applied to one end of the spool while the other end of the spool is vented to the atmosphere through a diaphragm assembly. When the valve moves up or down as directed by the imbalance between the diaphragm and spring forces, the valve spool either vents the positioner output port to the atmosphere or admits supply 


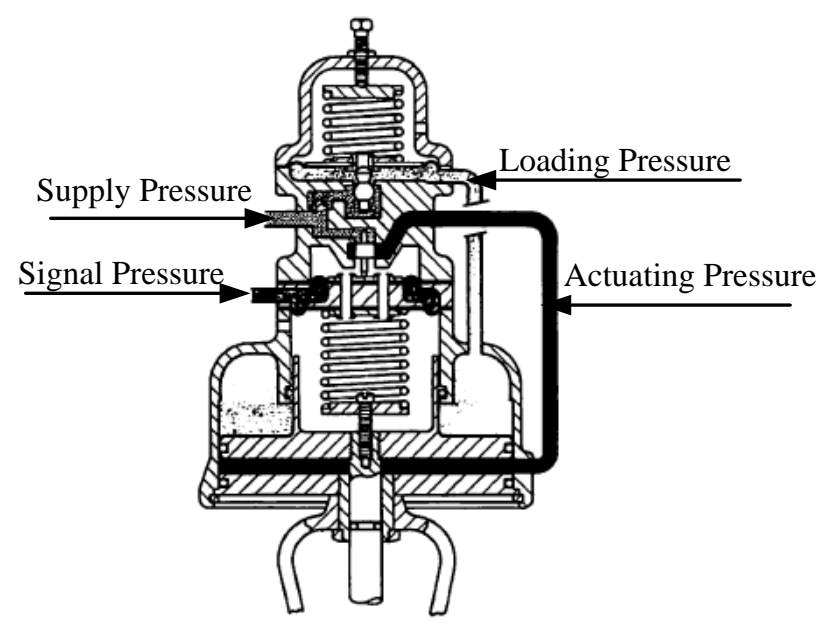

Fig. 3. Continuous controlled valve ([8])

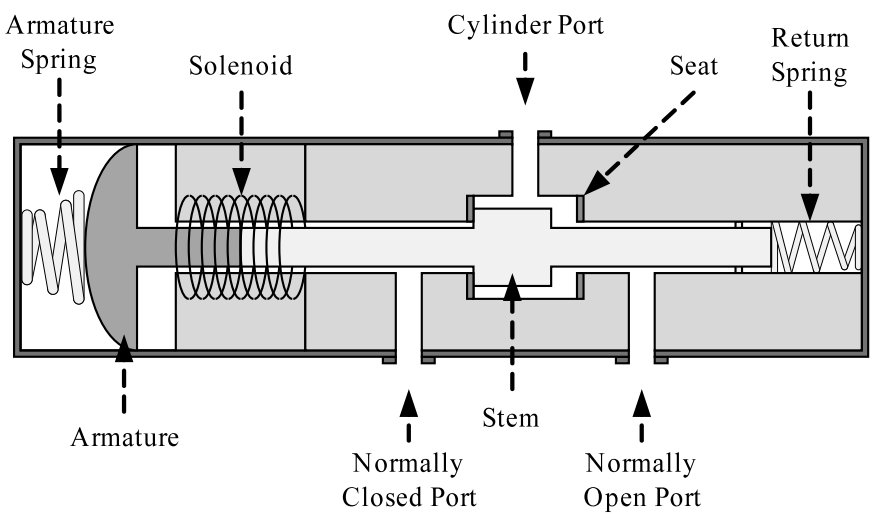

Fig. 4. Three-way two-position solenoid valve.

pressure to the positioner output port. Depending on the pilot valve position, the output pressure can vary from zero to full supply pressure, actuating the valve.

A three-way two-position solenoid valve (SV), illustrated in Figure 4, is used for controlling the operation of the DV valve. The cylinder port connects to the valve, the normally closed (NC) port connects to the supply pressure, and normally open (NO) port is left unconnected, allowing venting to atmosphere. When the solenoid is energized, the path from the NC port to cylinder port is open, allowing gas to pass from the supply to the valve, thus actuating the valve. When deenergized, the supply pressure is closed off and the path from the cylinder port to the NO port is opened, thus venting the DV valve which closes the valve due to the return spring. The solenoid is powered by $24 \mathrm{~V}$ DC either through the power supply or the Li-ion batteries for operation.

An IPT converts an analog current signal (4-20 mA) to a proportional linear pneumatic output ( 3 to $15 \mathrm{psig}$ ) and is used for controlling the operation of the CV valve. The IPT is essentially a pressure regulator that is controlled by a current signal. Supply pressure is connected to the input port, and, the regulated output pressure will change depending on the current signal.

Controllable gas leaks are introduced through a set of proportional valves (V1-V4). These valves combine a solenoid valve with an electronics package that digitally modulates the control signal to provide analog proportional control. These are two-way normally-closed valves and operate on $24 \mathrm{~V} \mathrm{DC}$, powered through the power supply or the batteries.

The data from the different sensors is collected using an 8slot NI cDAQ-9188 Gigabit Ethernet chassis which is designed for remote or distributed sensor and electrical measurements. In the experimental testbed, for safety requirements, all the controls and data acquisition activities are done remotely hence this specific chassis is selected for the testbed. A single NI CompactDAQ chassis can measure up to 256 channels of sensor signals, analog I/O, digital I/O, and counter/timers with an Ethernet interface back to a host machine. All the operations for the cDAQ-9188 are controlled through a LabVIEWdesigned interface from where the user can have access to all the input data and output control.

\section{System FAULTS}

As discussed earlier, the main focus of the prognostics testbed is to emulate valve faults in order to demonstrate remaining life prediction in the context of cryogenic refueling operations. The most common type of fault on the propellant loading testbed is a leak of pneumatic gas. To demonstrate valve prognostics for leaks, we have emulated faults at selected locations throughout the pressure distribution system where leaks are likely to appear. The fault injection is accomplished by adding a bypass line with a proportional solenoid valve in the pneumatic lines. The bypass valves are remotely operated and the position can be specified to control the leakage rate and support desired damage progression profiles. In addition, during certain experimental configurations, Li-ion batteries are used to power the solenoid valve. Faults are incorporated in the batteries to cause a premature loss of charge. Table I summarizes the different faults that may appear in the system and their effect on the components.

\section{A. Solenoid Valve and DV Leak Faults}

Figure 5 illustrates the setup for faults that can be injected in the solenoid valve when energized, to study the degradation effects on the operation of the DV. As illustrated, the leakage faults can be injected at the NO and NC seat ports using the bypass valve V1 which affects the DV operation due to decreased supply pressure. The leak through V1 emulates a leak at the cylinder port or, when energized, a leak across the NO seat (see Table I).

Similarly, Figure 6 illustrates the setup for faults that can be injected in the solenoid valve when de-energized, to study the degradation effects on the operation of the DV. A leakage fault can be injected at the NO and NC seat ports using the bypass valve V2 which affects the DV operation due to a decreased supply pressure. In both the injected faults, the amount the valve will open depends on steady-state pressure and whether it produces enough force to overcome the valve's return spring 
TABLE I

FAult InJeCtion Matrix For PROgnostics Testbed

\begin{tabular}{|c|c|c|c|}
\hline Component & Fault Mode & Effects & Injecting Component \\
\hline \multirow[t]{3}{*}{ Solenoid Valve } & Leak across NC seat & $\begin{array}{l}\text { If SV energized, and DV valve is open, no effect; if DV valve } \\
\text { is closed, no effect. If SV de-energized, and DV valve is closed, } \\
\text { DV valve potentially opens; if DV valve is open, DV closes more } \\
\text { slowly }\end{array}$ & $\mathrm{V} 2$ \\
\hline & Leak across NO seat & $\begin{array}{l}\text { If SV energized, and DV valve is open, loses pressure and DV can } \\
\text { start to close; if DV valve is closed, it will open more slowly. If } \\
\text { SV de-energized, and DV valve is closed, no effect; if DV valve } \\
\text { is open, will close more slowly }\end{array}$ & V1 \\
\hline & Leak at cylinder port & Same as leak across NC seat & $\mathrm{V} 2$ \\
\hline DV & Pneumatic gas leak at valve port & Same effects as leak at SV cylinder port or leak across NO seat & $\mathrm{V} 1$ \\
\hline IPT & Leak at output port & $\begin{array}{l}\text { Lowers regulated signal pressure which affects the open time of } \\
\text { the CV }\end{array}$ & $\mathrm{V} 3$ \\
\hline \multirow[t]{2}{*}{$\mathrm{CV}$} & Pneumatic gas leak at supply pressure port & $\begin{array}{l}\text { Lower supply pressure so valve may not open fully, open more } \\
\text { slowly }\end{array}$ & V4 \\
\hline & Pneumatic gas leak at signal pressure port & Lowers regulated pressure & V3 \\
\hline Li-ion Battery & Additional resistance & Reduced charge leaves the DV unable to actuate properly & $\mathrm{R} 1$ \\
\hline
\end{tabular}

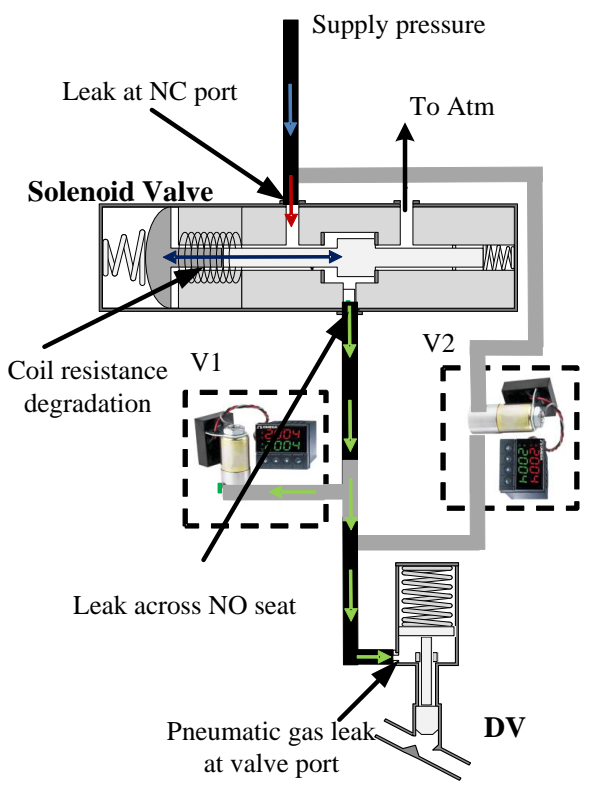

Fig. 5. Solenoid valve leak fault injection when energized on DV valve

force. The steady-state value and how long it takes to get there depends on the orifice size and leak rate. The amount the valve will close depends on steady-state pressure and the return spring force to overcome it.

\section{B. IPT and CV Valve Leak Faults}

Figure 7 illustrates the setup for faults that can be injected through the IPT and bypass valves, to study the degradation effects on the operation of the CV. As can be seen, a leakage

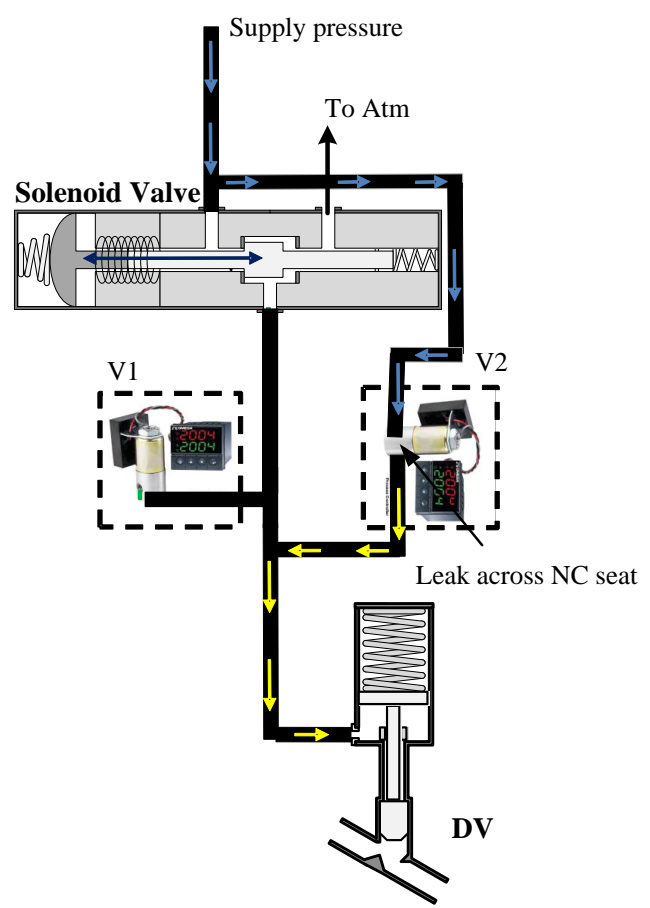

Fig. 6. Solenoid valve leak fault injection when de-energized on DV valve

fault can be injected at the supply pressure port through bypass valve V4. Any changes in the supply pressure will have an effect on the opening and closing of the CV valve. A leak in the signal pressure line from the IPT to the CV can also be injected through V3. Since the IPT modulates the amount of pressure at its output depending upon the control signal, a leak 


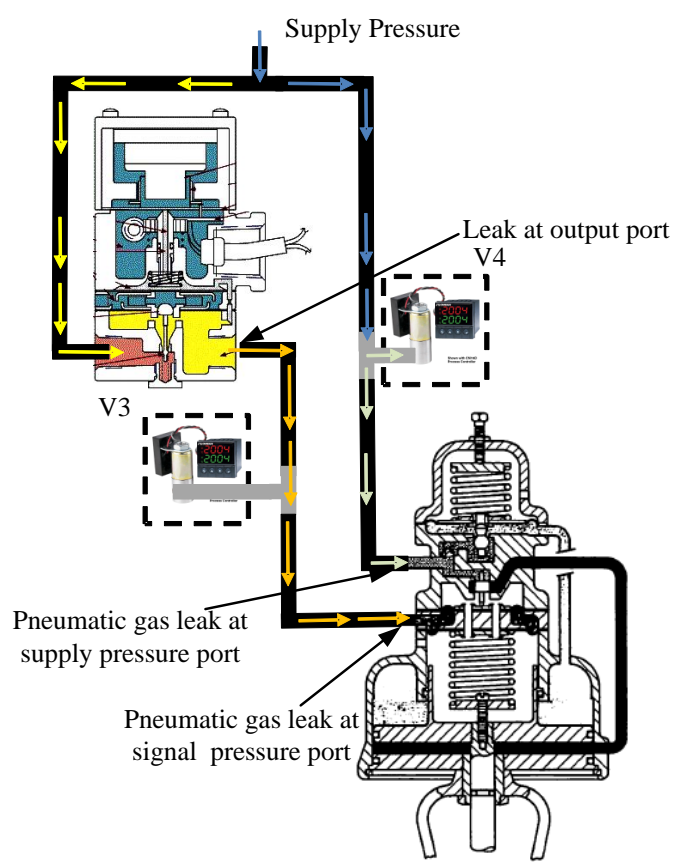

Fig. 7. CV pneumatic leak faults

on the signal line will reduce the signal pressure going to the $\mathrm{CV}$ and modify its position.

\section{Li-ion Batteries}

Li-ion batteries are used as an alternate power source to operate the solenoid valve. Over the period of operation the battery charge reduces and the batteries are considered to be fully discharged once they reach a certain threshold value. A completely charged battery cell has a maximum potential voltage of $4.2 \mathrm{~V}$. Six batteries in series are used to operate the solenoid valve. If the threshold for any of the 6 batteries falls below $2.6 \mathrm{~V}$, the solenoid valve connection is turned off, which, in turn, stops operating the DV valve. An resitance $\mathrm{R} 1$ was used in parallel to the SV as a load to discharge the batteries. The solenoid takes around $40 \mathrm{~mA}$ of during each operation which would take the batteries a very long time to drain. Hence to accentuate the drain process a $18 \mathrm{ohm}$ resistor $\mathrm{R} 1$ is added in parallel with the solenoid which drains the battery at a constant load of around 1.4 A to last for 100 cycles of operation of the DV valve.

\section{Integration with Prognostics Algorithms}

Each of the the faults discussed is exacuted independently in the system. For both the valves, changes in the opening and closing times are the parameters that change with leak faults. Hence, along with the pressure sensors to observe pressure changes, we use a LVDT sensor that is mounted on the shaft of each valve. In real systems only LVDT senosr data is availalbe and no pressure sensors are available. In this work the pressure sensors were used to observe the pressure changes to study the effects of faults in the systems. The prognostics algorithms use only LVDT data for making RUL and EOL predictios.
Changes in the opening and closing times help detect and isolate faults in the valves. For batteries, voltage, current, and temperature are the three meausrements that are used to estimate the state of charge (SOC). Experiments are controlled through the LABView front-end. Through LABView, sensor measurements are fed into the prognostics algorithms, implemented in Matlab. The prognostics algorithms compute health state estimates and life predictions.

\section{Prognostics Approach}

In this section we discuss the general prognosis framework used for the valves and batteries, following the general estimation-prediction framework of model-based prognostics [9], [10], [11]. Details of the specific algorithms are described in [5], [7] for the pneumatic valves, [12], [13] for the IPT, and [14] for the batteries. Here, we summarize the formulation the prognostics problem, followed by a brief description of the estimation approach and a description of the prediction approach.

\section{A. Problem Formulation}

We assume the system model may be generally defined as

$$
\begin{aligned}
\mathbf{x}(k+1) & =\mathbf{f}(k, \mathbf{x}(k), \boldsymbol{\theta}(k), \mathbf{u}(k), \mathbf{v}(k)), \\
\mathbf{y}(k) & =\mathbf{h}(k, \mathbf{x}(k), \boldsymbol{\theta}(k), \mathbf{u}(k), \mathbf{n}(k)),
\end{aligned}
$$

where $k$ is the discrete time variable, $\mathbf{x}(k) \in \mathbb{R}^{n_{x}}$ is the state vector, $\boldsymbol{\theta}(k) \in \mathbb{R}^{n_{\theta}}$ is the unknown parameter vector, $\mathbf{u}(k) \in$ $\mathbb{R}^{n_{u}}$ is the input vector, $\mathbf{v}(k) \in \mathbb{R}^{n_{v}}$ is the process noise vector, $\mathbf{f}$ is the state equation, $\mathbf{y}(k) \in \mathbb{R}^{n_{y}}$ is the output vector, $\mathbf{n}(k) \in \mathbb{R}^{n_{n}}$ is the measurement noise vector, and $\mathbf{h}$ is the output equation. ${ }^{1}$

In prognostics, we predict the occurrence of an event $E$ that is defined with respect to the states, parameters, and inputs of the system. We define the event as the earliest instant that some event threshold $T_{E}: \mathbb{R}^{n_{x}} \times \mathbb{R}^{n_{\theta}} \times \mathbb{R}^{n_{u}} \rightarrow \mathbb{B}$, where $\mathbb{B} \triangleq\{0,1\}$ changes from the value 0 to 1 . That is, the time of the event $k_{E}$ at some time of prediction $k_{P}$ is defined as

$$
k_{E}\left(k_{P}\right) \triangleq \inf \left\{k \in \mathbb{N}: k \geq k_{P} \wedge T_{E}(\mathbf{x}(k), \boldsymbol{\theta}(k), \mathbf{u}(k))=1\right\} .
$$

The time remaining until that event, $\Delta k_{E}$, is defined as

$$
\Delta k_{E}\left(k_{P}\right) \triangleq k_{E}\left(k_{P}\right)-k_{P} \text {. }
$$

For systems health management, $T_{E}$ is defined via a set of performance constraints that define what the acceptable states of the system are, based on $\mathbf{x}(k), \boldsymbol{\theta}(k)$, and $\mathbf{u}(k)$ [11]. For valves, timing requirements are provided that define the maximum allowable time a valve may take to open or close [4]. For batteries, we are interested in EOD, defined by a voltage threshold $V_{E O D}$.

Models of the system components are constructed in this paradigm that capture both nominal behavior, as well as faulty behavior and damage progression. Using these models, observations can be mapped back to the health state of the system as represented in $\mathbf{x}$ and $\boldsymbol{\theta}$. An estimation algorithm,

\footnotetext{
${ }^{1}$ Bold typeface denotes vectors, and $n_{a}$ denotes the length of a vector $\mathbf{a}$.
} 
Prognostics

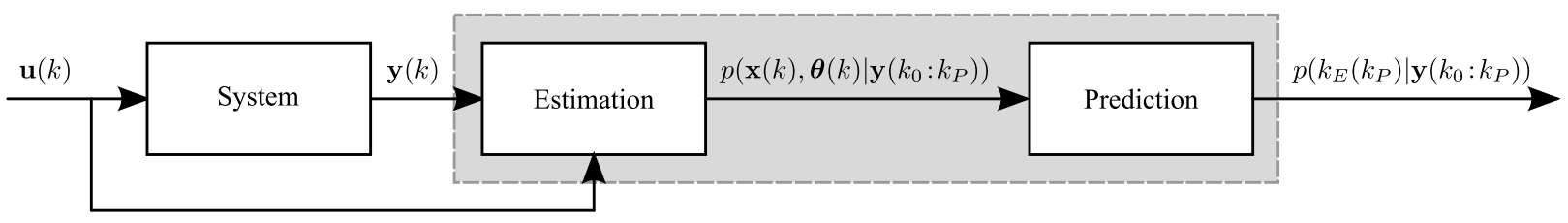

Fig. 8. Prognostics architecture.

such as the Kalman filter, unscented Kalman filter, or particle filter, is used to solve this problem [15]. This state-parameter estimate, along with a prediction of the future usage of the component, is used as input to a prediction algorithm that computes EOL and remaining useful life (RUL) [11], [16]. In order to account for fault effects propagating across different components, such as a leak through a solenoid valve causing changes in pneumatic valve behavior, a system-level perspective is needed for prognostics [17].

\section{B. Prognostics Architecture}

In a model-based prognostics architecture [11], there are two sequential problems, (i) the estimation problem, which requires determining a joint state-parameter estimate $p\left(\mathbf{x}(k), \boldsymbol{\theta}(k) \mid \mathbf{y}\left(k_{0}: k\right)\right)$ based on the history of observations up to time $k, \mathbf{y}\left(k_{0}: k\right)$, and (ii) the prediction problem, which determines at $k_{P}$, using $p\left(\mathbf{x}(k), \boldsymbol{\theta}(k) \mid \mathbf{y}\left(k_{0}: k\right)\right)$, a probability distribution $p\left(k_{E}\left(k_{P}\right) \mid \mathbf{y}\left(k_{0}: k_{P}\right)\right)$. The distribution for $\Delta k_{E}$ can be trivially computed from $p\left(k_{E}\left(k_{P}\right) \mid \mathbf{y}\left(k_{0}: k_{P}\right)\right)$ by subtracting $k_{P}$.

The prognostics architecture is shown in Fig. 8 [11]. In discrete time $k$, the system is provided with inputs $\mathbf{u}_{k}$ and provides measured outputs $\mathbf{y}_{k}$. The estimation module uses this information, along with the system model, to compute an estimate $p\left(\mathbf{x}(k), \boldsymbol{\theta}(k) \mid \mathbf{y}\left(k_{0}: k\right)\right)$. The prediction module uses the joint state-parameter distribution and the system model, along with hypothesized future inputs, to compute the probability distribution $p\left(k_{E}\left(k_{P}\right) \mid \mathbf{y}\left(k_{0}: k_{P}\right)\right)$ at given prediction times $k_{P}$.

\section{Estimation}

For both the valves and the batteries, we developed a detailed physics model of component behavior using nominal data from the testbed which is discussed in [7] and [14] respectively. For the valves, we then simulate for various leakage rates, computing the corresponding open and close times. This information is encoded in a lookup table, so, given measured open and close times, we can quickly obtain the corresponding leakage rate. For the batteries, we use an unscented Kalman filter (UKF) to obtain the state estimate from the sensor measurements, as described in [14].

\section{Prediction}

For the valves, from estimation we have estimated leakage rates under different fault assumptions. We can then project out the leakage rates to determine the time at which they reach values corresponding to EOL, assuming a progression profile. Different faults have different effects on open and close times, allowing to distinguish which fault is present. Predictions are made once the measured valve timing exceeds specified nominal thresholds.

For the batteries, we simulate for various SOC values and load values the corresponding remaining time until discharge, and compuate a lookup table. Given the SOC, as computed by the UKF, and expected future load, we can then quickly compute the corresponding time of EOD.

\section{EXPERIMENTS AND RESULTS}

We present here results observed for the fault injection experiments conducted using the prognostic testbed. In each of the experiment the fault is injected into one of the components until the fault progression reaches its maximum extent, i.e., the component reaches its end of life condition.

\section{A. Leak to Atmosphere : DV Fault}

The leak to atmosphere fault is injected by controlling the position of the leak valve V1. Faults in the valves are injected by linearly increasing the open percentage of the desired leak valve in increments of $1 \%$. This emulates a leak across the NO seat of the solenoid valve, or a leak on the gas line going to the pneumatic valve. This fault causes a decrease in opening times and an increase in closing times. Fig. 9 shows the open times of the valve during the fault progression, and Fig. 10 shows the close times. It is difficult to determine a trend in the open times, and they do not cross the detection threshold. The close times are very noisy, and typically cross the closing time threshold at the 48th cycle. Based on the open and close times, the fault must be a leak to atmosphere, in agreement with the model.

\section{B. Leak from Supply: DV Fault}

As described in Section II, the leak from supply fault is injected by controlling the position of the leak valve V2. This emulates a leak across the NC seat of the solenoid valve. This fault causes an increase in opening times and a slight decrease in closing times. Fig. 11 shows the open times of the valve during the fault progression, and Fig. 12 shows the close times. The observed trends are in agreement with the model. A fault is detected at the 43rd cycle based on the opening times.

\section{Leak from Signal: CV Fault}

The leak from signal fault is injected by controlling the position of the leak valve V3. This fault emulates a leak a leak at the output port of the IPT or leak at the input port of 


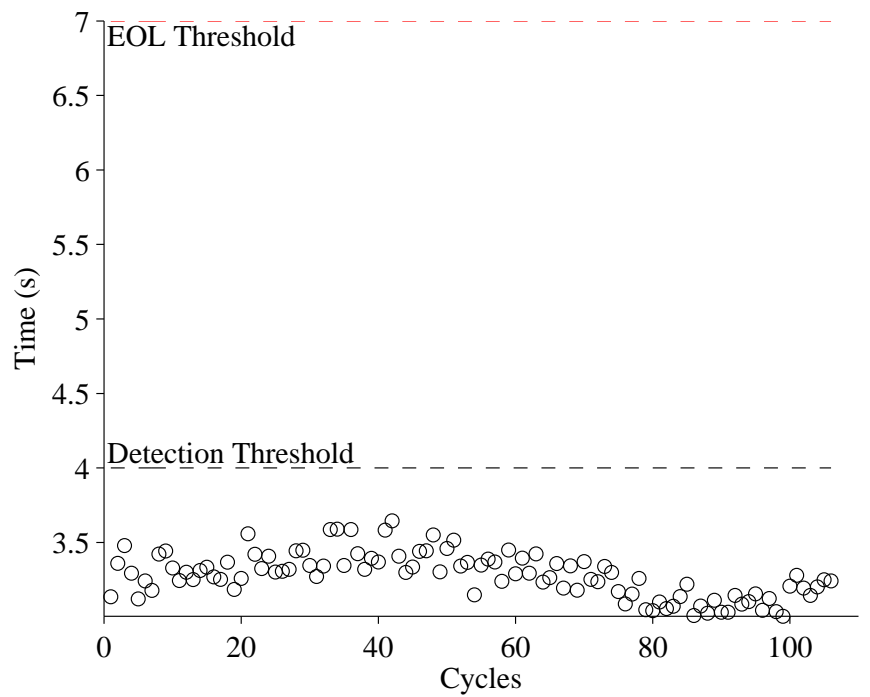

Fig. 9. DV open times with a atmosphere leak.

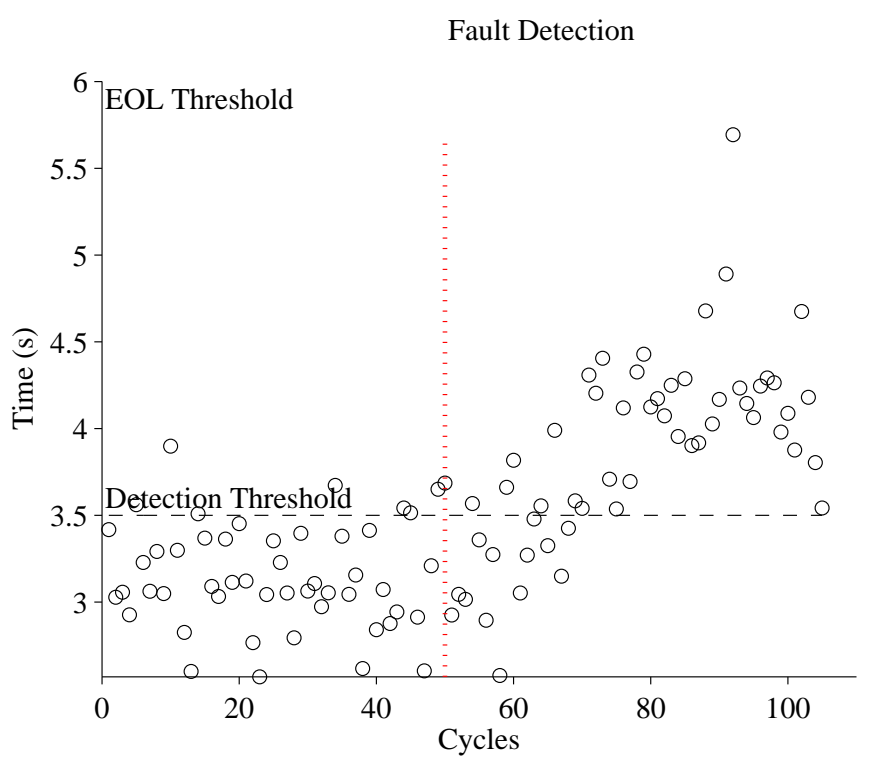

Fig. 10. DV close times with a atmosphere leak.

the CV valve. This fault causes a increase in the opening time as shown in Fig.13 while the close time does not change. It is also observed from Fig. 14 that the steady state value of the $\mathrm{CV}$ decreases in this fault. It is difficult to determine a trend in the close times, and they do not cross the detection threshold. Since nominal open times are noisy a threshold of $7.6 \mathrm{sec}$ is set with a mean of last 3 values. A threshold of 0.997 for steady state percent values is set to detect a fault in the operation of the valve.

\section{Leak from Supply: CV Fault}

The leak from supply fault is injected by controlling the position of the leak valve V4. This fault emulates a leak in the supply line or the supply input port of the CV. This fault causes a increase in the opening time as shown in Fig. 15

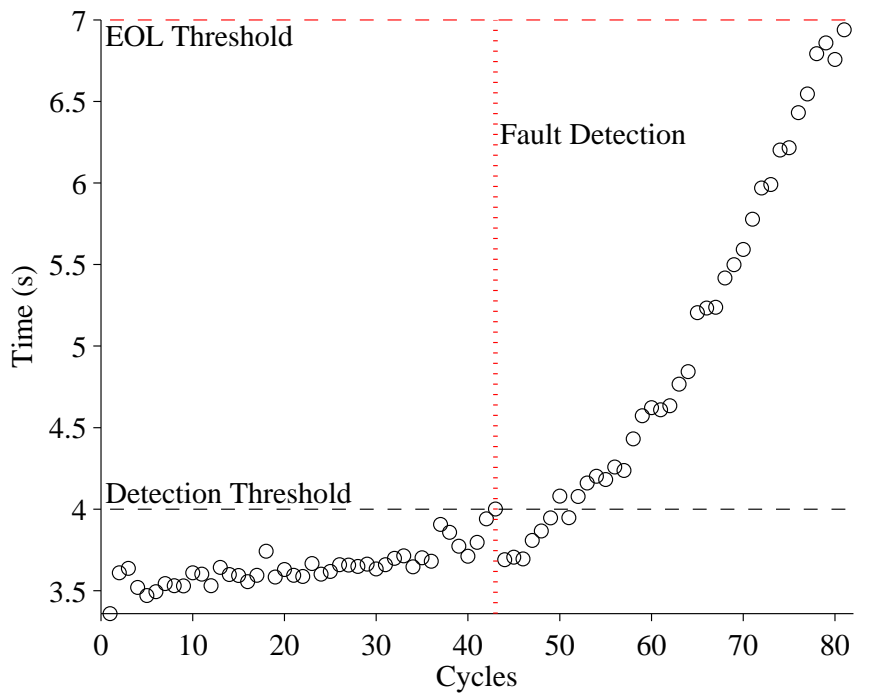

Fig. 11. DV open times with a leak from supply.

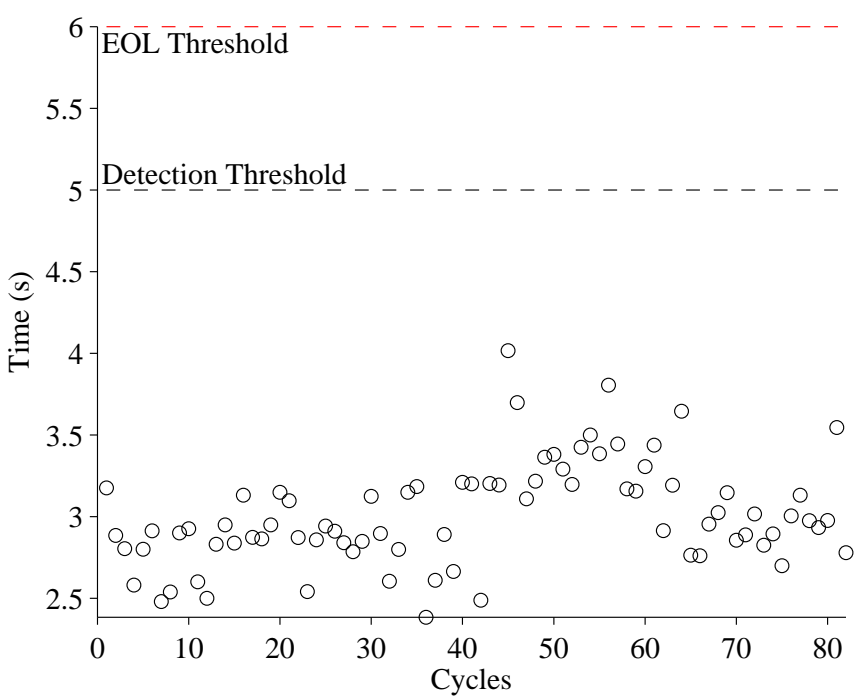

Fig. 12. DV close times with a leak from supply.

while the close time does not change. It is also observed from Fig. 16 that the steady state value of the $\mathrm{CV}$ decreases in this fault. It is difficult to determine a trend in the close times, and they do not cross the detection threshold. Since nominal open times are noisy a threshold of $7.6 \mathrm{sec}$ is set with a mean of last 3 values.

\section{E. Battery Degradation Fault}

As mentioned earlier in Section II, 6 batteries with a combined voltage of around $24.5 \mathrm{~V}$ are used to power the solenoid operating the DV. Fig. 17 shows the discharge cycle for one of the batteries reaching the threshold voltage.

We discuss the prognostics results based on the experiments conducted using the derived model. We use the architecture described in [14]. To acheive accurate prognostics results the developed model should be accurate which in this case is the 


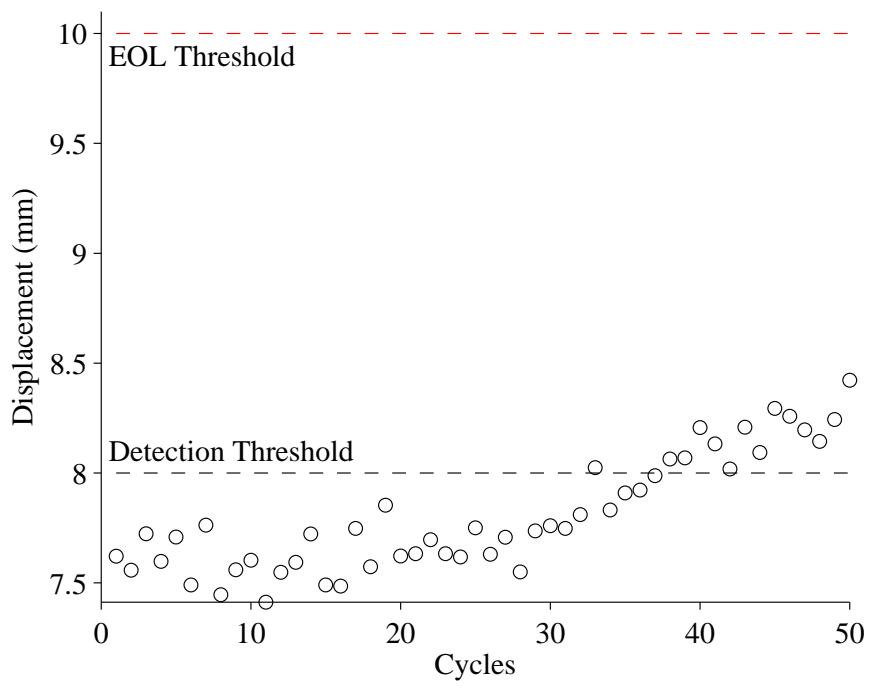

Fig. 13. CV open times with a signal leak.

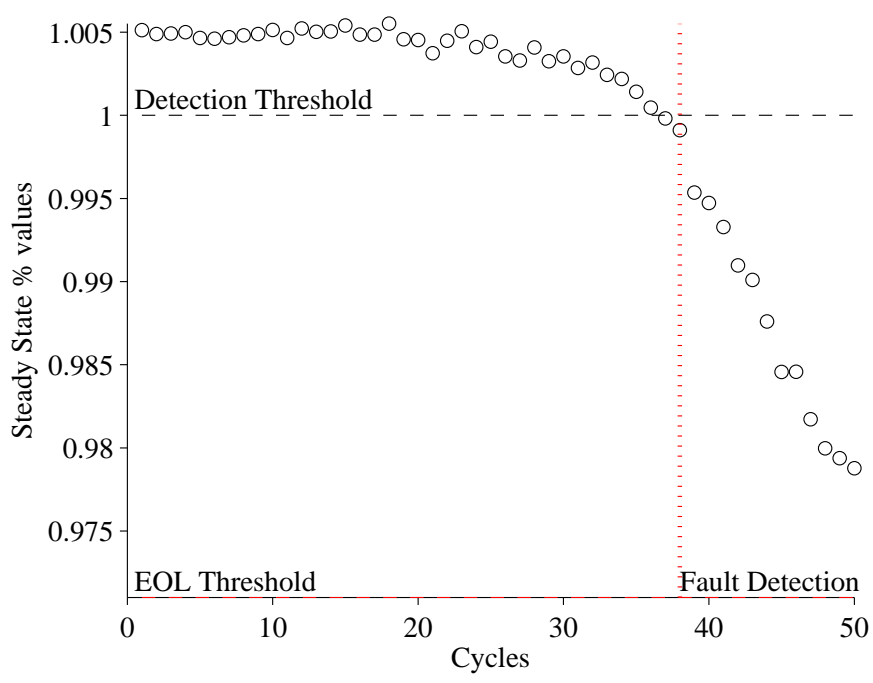

Fig. 14. CV steady state position times with a signal leak.

electrochemistry Li-ion battery model [14] along with known future inputs.

Fig.18 shows the plots for state of charge (SOC) for all the 6 batteries and Fig.19 shows the voltage drop due to load till one of the batteries reaches the cutoff voltage of $2.6 \mathrm{~V}$. The experiment was stopped when any one of the batteries reached a threshold of $2.6 \mathrm{~V}$ which avoided the batteries going into deep discharge.

We plot results in $\alpha-\lambda$ plots, where $\alpha$ (e.g. 10\%) defines an accuracy cone around the ground truth, and $\lambda$ is a time point [20]. As seen from the RUL plot in Fig.20 it is observed that the prediction during discharge cycle experiment is within the $\alpha-\lambda$ cone. This indicates the model is predicting EOL with high accuracy.

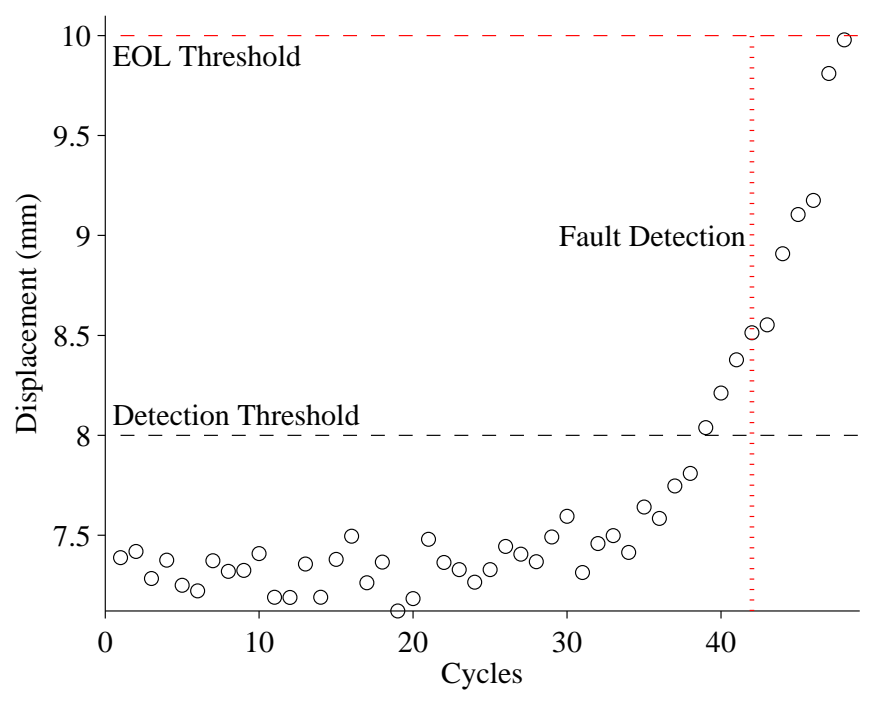

Fig. 15. CV open times with a supply leak.

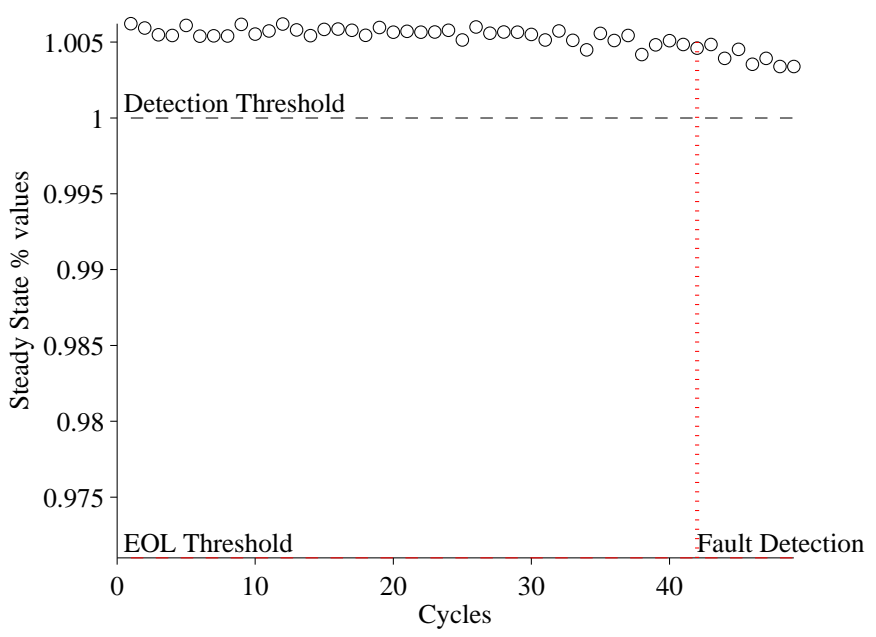

Fig. 16. CV stady state position times with a supply leak.

\section{CONCLUSION}

In this paper, we presented a prognostics demonstration testbed for pneumatic valves and batteries in the context of cryogenic propellant loading systems. We injected controlled faults and collected data in the system using the developed hardware interface. This data was used to develop component models and to implement prognostic algorithms such that we are able to make accurate EOL predictions. The testbed helps in studying relalistic degradation phenomenona and failure effects in the different components of propellant loading systems. The study will help us implement the developed prognostic methodologies in the field and aid operations crew to make effective maintenance-related decisions.

In the current testbed we able to inject few major faults since over the period of time through the experiments we have found that the operational relationship between the injected faults and the components is very complicated. We are currently 


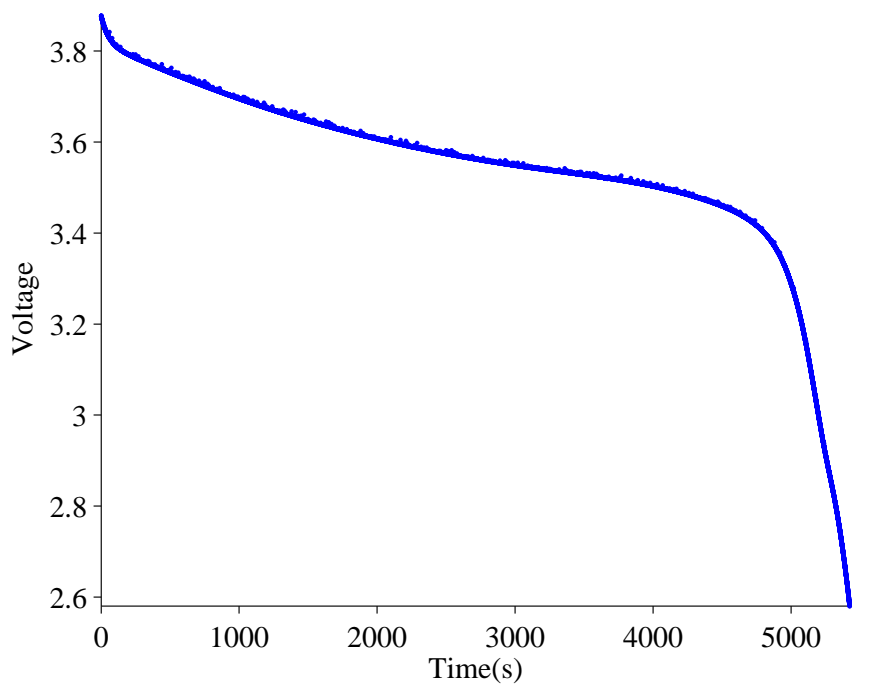

Fig. 17. Voltage discharge for battery 4

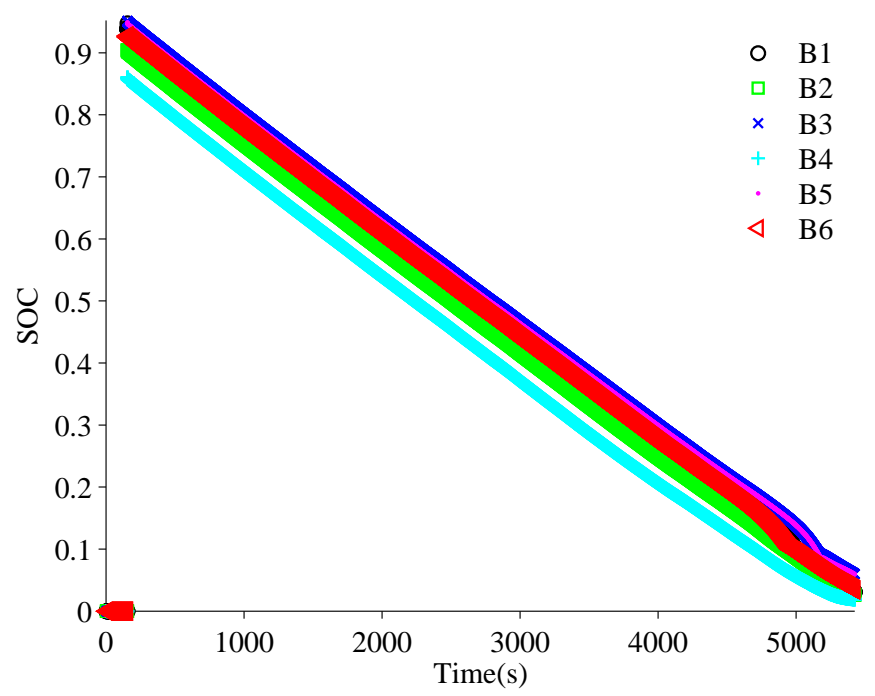

Fig. 18. Estimated SOC for all 6 batteries

studying more faults that could be injected as well as detected and isolated using our algorithms. As we better understand the system and are able to model the system better, we can incorporate those faults. The developed testbed was integrated with the field system to conduct similar experiments, for which the analysis and results are part of our future work.

\section{ACKNOWLEDGMENT}

This work was funded by the Advanced Ground Systems Maintenance (AGSM) Project under the Ground Systems Development and Operations program.

\section{REFERENCES}

[1] N. P. Zeitlin, G. R. Clements, S. J. Schaefer, M. K. Fawcett, and B. L. Brown, "Ground and launch systems processing technologies to reduce overall mission life cycle cost," in 2013 IEEE Aerospace Conference, Mar. 2013.

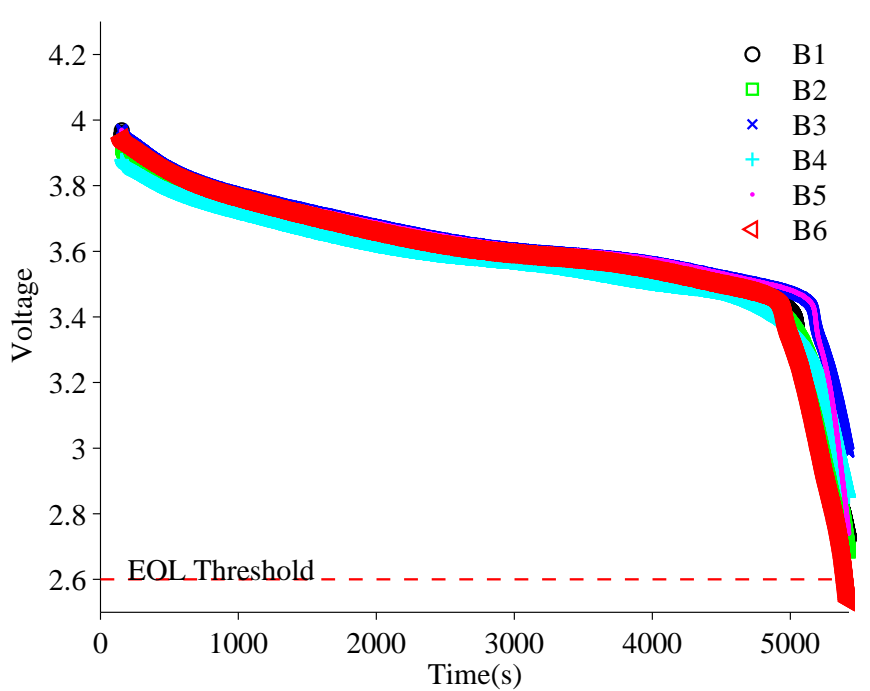

Fig. 19. Estimated Voltage for all 6 batteries

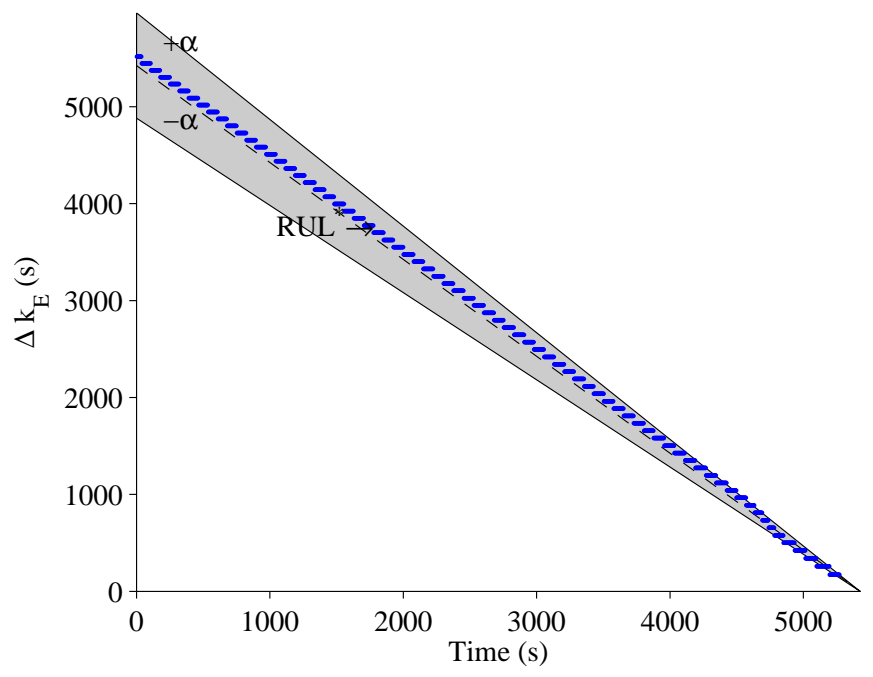

Fig. 20. RUL prediction for Battery 4

[2] C. Goodrich, S. Narasimhan, M. Daigle, W. Hatfield, R. Johnson, , and B. Brown, "Applying model-based diagnosis to a rapid propellant loading system," in Proceedings of the 20th International Workshop on Principles of Diagnosis, Jun. 2009, pp. 147-154.

[3] J. Barber, K. Johnston, and M. Daigle, "A cryogenic fluid system simulation in support of integrated systems health management," in Annual Conference of the Prognostics and Health Management Society 2013, Oct. 2013, pp. 114-126.

[4] M. Daigle and K. Goebel, "A model-based prognostics approach applied to pneumatic valves," International Journal of Prognostics and Health Management, vol. 2, no. 2, Aug. 2011.

[5] M. Daigle, C. Kulkarni, and G. Gorospe, "Application of model-based prognostics to a pneumatic valves testbed," in Proceedings of the 2014 IEEE Aerospace Conference, Mar. 2014.

[6] C. Kulkarni, M. Daigle, and K. Goebel, "Implementation of prognostic methodologies to cryogenic propellant loading testbed," in IEEE $A U$ TOTESTCON 2013, Sep. 2013.

[7] C. Kulkarni, M. Daigle, G. Gorospe, and G. Goebel, "Validation of model-based prognostics for pneumatic valves in a demonstration testbed," in Annual Conference of the Prognostics and Health Management Society 2014, Oct. 2014.

[8] "Er4000 masoneilan domotor actuator instructions," 1991, rev. B8/84. 
[9] J. Luo, K. R. Pattipati, L. Qiao, and S. Chigusa, "Model-based prognostic techniques applied to a suspension system," IEEE Transactions on Systems, Man and Cybernetics, Part A: Systems and Humans, vol. 38, no. 5, pp. $1156-1168$, Sep. 2008.

[10] M. Orchard and G. Vachtsevanos, "A particle filtering approach for online fault diagnosis and failure prognosis," Transactions of the Institute of Measurement and Control, no. 3-4, pp. 221-246, Jun. 2009.

[11] M. Daigle and K. Goebel, "Model-based prognostics with concurrent damage progression processes," IEEE Transactions on Systems, Man, and Cybernetics: Systems, vol. 43, no. 4, pp. 535-546, May 2013.

[12] C. Teubert and M. Daigle, "I/P transducer application of modelbased wear detection and estimation using steady state conditions," in Proceedings of the Annual Conference of the Prognostics and Health Management Society 2013, Oct. 2013, pp. 134-140.

[13] _ , "Current/pressure transducer application of model-based prognostics using steady state conditions," in 2014 IEEE Aerospace Conference, Mar. 2014.

[14] M. Daigle and C. Kulkarni, "Electrochemistry-based battery modeling for prognostics," in Annual Conference of the Prognostics and Health Management Society 2013, Oct. 2013, pp. 249-261.
[15] M. Daigle, B. Saha, and K. Goebel, "A comparison of filter-based approaches for model-based prognostics," in Proceedings of the 2012 IEEE Aerospace Conference, Mar. 2012.

[16] M. Daigle, A. Saxena, and K. Goebel, "An efficient deterministic approach to model-based prediction uncertainty estimation," in Annual Conference of the Prognostics and Health Management Society, Sep. 2012, pp. 326-335.

[17] M. Daigle, A. Bregon, and I. Roychoudhury, "A distributed approach to system-level prognostics," in Annual Conference of the Prognostics and Health Management Society, Sep. 2012, pp. 71-82.

[18] S. J. Julier and J. K. Uhlmann, "A new extension of the Kalman filter to nonlinear systems," in Proceedings of the 11th International Symposium on Aerospace/Defense Sensing, Simulation and Controls, 1997, pp. 182193.

[19] - "Unscented filtering and nonlinear estimation," Proceedings of the IEEE, vol. 92, no. 3, pp. 401-422, Mar 2004.

[20] A. Saxena, J. Celaya, B. Saha, S. Saha, and K. Goebel, "Metrics for offline evaluation of prognostic performance," International Journal of Prognostics and Health Management, vol. 1, no. 1, 2010. 